\title{
Capillary blood flow in the canine colon and other organs at normal and raised portal pressure
}

\author{
A S GRANDISON, J YATES, AND R SHIELDS \\ From the Department of Surgery, University of Liverpool, Liverpool
}

SUMMARY Capillary blood flow in the canine colon and other organs has been assessed over a wide range of portal pressures using radio-labelled microspheres. The proximal colon received a greater proportionate flow than the distal region. At both normal and raised portal pressures, the mucosa received $90 \%$ of the total colonic capillary flow. A highly significant negative correlation $(\mathrm{P}<0.001)$ was found between colonic capillary blood flow and portal pressure. A highly significant positive correlation $(\mathrm{P}<0.001)$ was found between colonic capillary blood flow and flow in the superior mesenteric artery.

We have recently reported a clear relationship between portal pressure and absorption of ammonia, water, and electrolytes from the canine colon over a wide range of portal pressures. ${ }^{1}$ As portal pressure increased, absorption of ammonia, water, sodium, and chloride was reduced, and this reduction was abolished when portal pressure was allowed to return to the basal level. It seems likely that increased portal pressure, which reduces splanchnic flow, ${ }^{1}$ also slows mucosal capillary blood flow. As a result, the concentration gradient across the mucosa is diminished and so rates of passive intestinal transport will be decreased.

In these studies we have attempted to define the relationship between portal pressure and colonic mucosal capillary blood flow using radio-labelled microspheres. In addition, we have investigated the relative capillary blood flows to different regions of the intestine and other organs.

\section{Methods}

\section{ANIMALS}

Mongrel dogs (weight $15-20 \mathrm{~kg}$ ) were maintained under general anaesthesia throughout all surgical procedures and injection of microspheres.

\section{SURGICAL PROCEDURES}

With the animal lying supine, the abdomen was opened through a midline incision. A Dunn Vessel Occluder (J G Franklin Ltd), was placed around the portal vein, a flow probe was fitted around the

Received for publication 22 October 1980. superior mesenteric artery, and a cannula was inserted into a proximal mesenteric branch of the portal vein. Portal pressure was varied by inflation or deflation of the pneumatic cuff of the vessel occluder. Portal venous pressure and splanchnic blood flow were measured as described previously. ${ }^{1}$

An angiographic catheter was inserted into the right femoral artery and manipulated so that the tip was inside the left ventricle. The position of the catheter tip was confirmed radiologically.

The left femoral artery was cannulated to measure systemic arterial pressure.

A period of one hour with the vessel occluder completely deflated was allowed before the experiments started.

\section{PREPARATION OF MICROSPHERES}

Microspheres labelled with ${ }^{51} \mathrm{Cr},{ }^{141} \mathrm{Ce}$ and ${ }^{85} \mathrm{Sr}$ were obtained from the $3 \mathrm{M} \mathrm{Co}$. Ltd. The diameters of 100 of each type of microsphere were measured using a microscope with eye-piece graticule and were found to be $14 \cdot 20 \pm 0 \cdot 10 \mu, 13 \cdot 88 \pm 0 \cdot 10 \mu$, and $14 \cdot 50 \pm 0 \cdot 16 \mu$ respectively (mean \pm SEM).

Samples of 100-200 microspheres were placed on small strips of graph paper and the numbers exactly counted using an operating microscope. This was performed five times for each variety of microsphere. The strips were transferred to counting tubes, and by radioactive counting of the tubes on the day of each experiment, the specific activity of the microspheres (cpm/microsphere) was computed.

Samples for injection (approximately 400000 microspheres) were exactly measured using a Phillips PW 4580 automatic gamma analyser and 
made up to $5 \mathrm{ml}$ with $0.9 \%$ (w/v) sodium chloride solution containing $0.02 \%(\mathrm{v} / \mathrm{v})$ Tween 80 . At the end of each experiment, syringes and containers of the injection samples were counted for the appropriate isotope and the values were subtracted from the measured dose.

\section{INJECTION OF MICROSPHERES}

Immediately before injection the samples were shaken and placed in a sonicator bath for two minutes to disaggregate the microspheres. The samples were steadily injected with a $5 \mathrm{ml}$ syringe through the angiographic catheter into the left ventricle over a period of five seconds. Immediately after each injection, $5 \mathrm{ml}$ saline solution (containing $0.02 \%$ Tween 80 ) were injected to rinse the syringe and catheter.

\section{EXPERIMENTAL PROTOCOL}

(1) In two dogs (1-2), the angiographic catheter was introduced as described above, but no other surgical procedure was performed. ${ }^{85} \mathrm{Sr}$-labelled microspheres were injected into the left ventricle and after 15 minutes the animal was killed. The entire colon was removed, opened along its antimesenteric border, and thoroughly cleaned with $0.9 \%(w / v)$ sodium chloride solution. The colon was dried with tissue paper and the serosal layer was completely stripped off with forceps. The mucosa was then pulled away from the submucosa in one piece and the three tissues were weighed, cut into strips, and counted for ${ }^{85} \mathrm{Sr}$ activity.

In another two dogs (3-4) this procedure was repeated with the vessel occluder inflated so that the portal pressure was $35 \mathrm{mmHg}$ at the time of injection.

(2) In eight dogs (5-12) the entire surgical procedure described above was performed. The three types of microsphere were injected into the left ventricle at three different portal pressures including basal portal pressure (with the vessel occluder completely deflated). The portal pressure and flowmeter readings at the time of injection were recorded. Each injection was performed only when a steady portal pressure had been maintained for 10 minutes, and a period of 10 minutes was allowed before another adjustment of the vessel occluder was made. The order in which the types of microsphere were injected and the order in which the pressure adjustments were made were both randomised. In two of the dogs only two injections were possible.

The animals were killed 10 minutes after the final injection and the entire colon was removed, cleaned, cut into strips, and weighed. In six of the dogs (7-12) the colon was first cut into five equal length pieces from proximal to distal ends before further cutting and placing into counting tubes. Duplicate samples of ileum, jejunum, duodenum, stomach, liver, kidney, lung, and spleen were removed, cleaned, and weighed.

\section{RADIOACTIVE COUNTING}

Tissue samples were counted for 10 minutes in a Phillips PW450 automatic gamma analyser using preset window selectors corresponding to the three isotopes.

Samples of approximately $100000 \mathrm{cpm}$ of the three isotopes were counted using each of the three window selectors. Equations were thus constructed to calculate the actual cpm of each isotope present in the tissue samples from the counts measured in each window, when more than one isotope was present.

\section{CALCULATION OF RESULTS AND}

STATISTICAL ANALYSIS

(1) In dogs (1-4) the number of microspheres retained in each colonic layer was calculated and expressed as a percentage of the total number of microspheres recovered in the colon.

(2) In dogs (5-12) the number of each type of microsphere in each tissue sample was calculated and relative capillary flow was expressed as \% total injected/g intestine. For the colon this value represents the mean for the entire organ but for other tissues it is the mean of duplicate samples. The results were plotted against the appropriate portal pressure or mesenteric blood flow readings for each dog and subjected to analysis of covariance. ${ }^{2}$ In dogs (7-12) the relative capillary flow in each of the five regions of the colon was similarly computed and compared by paired $t$ test.

\section{Results}

The relative capillary blood flows to the three layers of the colon were assessed at basal portal pressure (dogs 1-2) and at a raised portal pressure of 35 $\mathrm{mmHg}$ (dogs 3-4). At basal portal pressure more than $90 \%$ of the colonic capillary blood flow was in the mucosa, the submucosa receiving approximately twice that of the serosal layer (Table). This distribution of capillary flow to the three layers was not changed at raised portal pressure.

Table Relative capillary blood flow to the three colonic layers

\begin{tabular}{|c|c|c|c|c|}
\hline & \multicolumn{2}{|c|}{ Basal portal pressure } & \multicolumn{2}{|c|}{ Raised portal pressure } \\
\hline & $\begin{array}{l}\text { Capillary flow } \\
(\% \text { total colon })\end{array}$ & $\begin{array}{l}W t \\
(g)\end{array}$ & $\begin{array}{l}\text { Capillary flow } \\
(\%) \text { total colon })\end{array}$ & $\begin{array}{l}W t \\
(g)\end{array}$ \\
\hline $\begin{array}{l}\text { Mucosa } \\
\text { Submucosa } \\
\text { Serosa }\end{array}$ & $\begin{array}{r}92 \cdot 2 \\
5 \cdot 5 \\
2 \cdot 3\end{array}$ & $\begin{array}{l}21 \cdot 4 \\
19 \cdot 8 \\
23 \cdot 5\end{array}$ & $\begin{array}{r}90 \cdot 3 \\
7 \cdot 1 \\
2 \cdot 6\end{array}$ & $\begin{array}{l}19 \cdot 6 \\
18 \cdot 4 \\
20 \cdot 1\end{array}$ \\
\hline
\end{tabular}

All values: mean of two experiments. 
Capillary blood flow in the canine colon

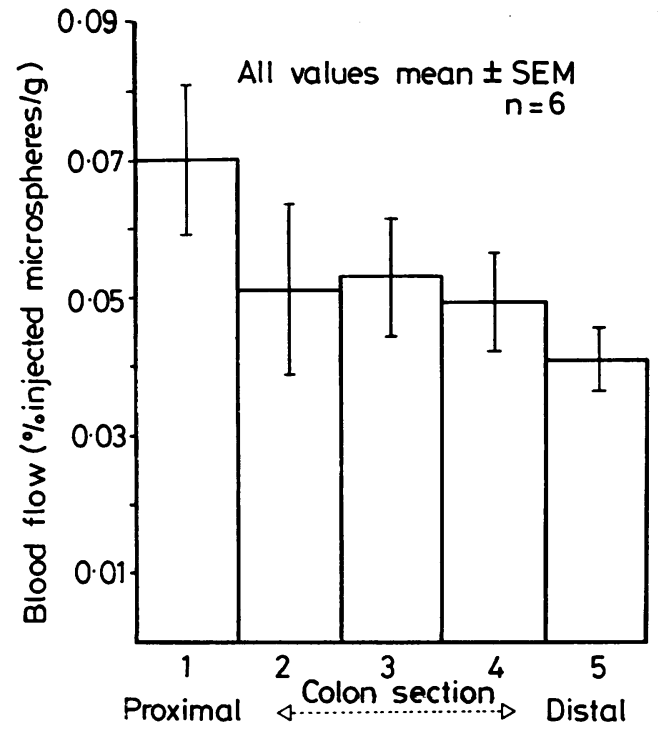

Fig. 1 Regional variation in capillary blood flow in the colon (dogs 7-12).

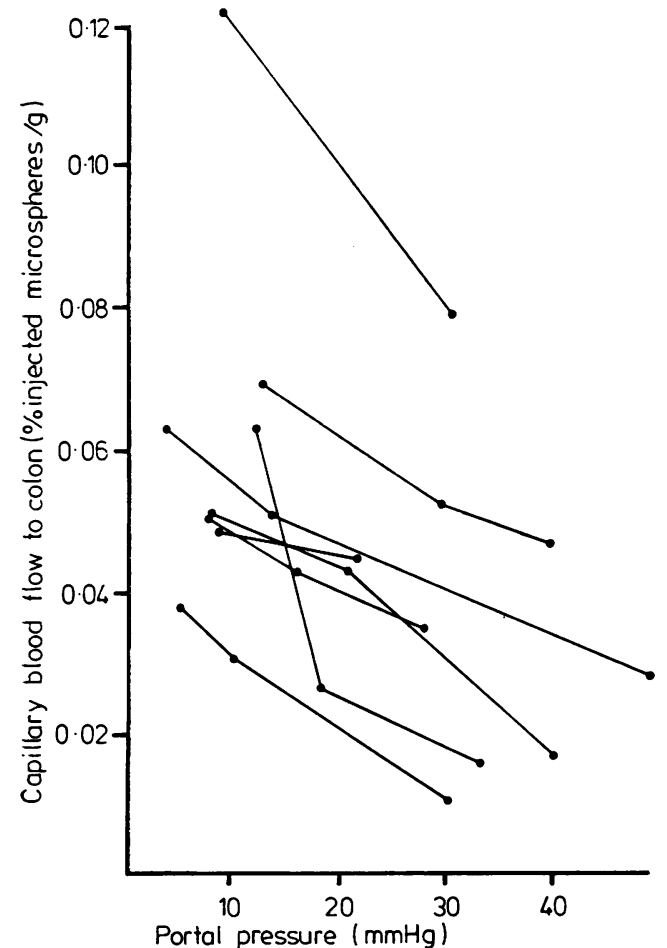

Fig. 2 The relationship between colonic capillary blood flow and portal pressure (dogs 5-12).

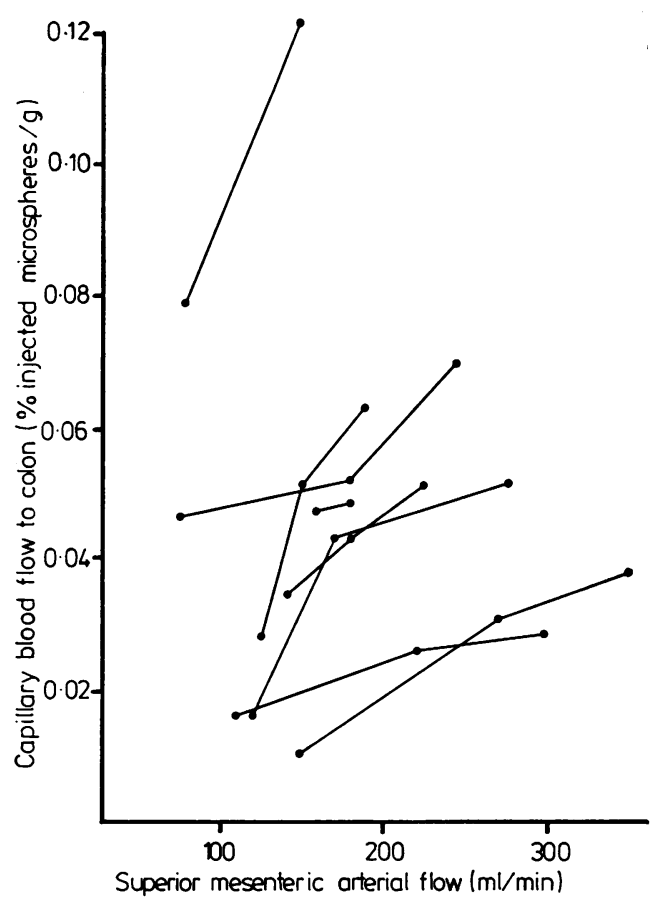

Fig. 3 The relationship between colonic capillary blood and superior mesenteric arterial flow at varying portal pressures (dogs 5-12).



Fig. 4 The relative capillary blood flows in various organs of the dog (dogs 5-12). 
The distribution of capillary flow along the length of the colon was assessed by dividing the colon into five sections of equal length and considering each section separately (dogs 7-12). A progressive fall in capillary flow from proximal to distal regions is apparent (Fig. 1), although the values for the extreme sections ( 1 and 5 ) were the only ones to display statistically significant difference $(t=3.44 ; \mathrm{P}<0.05)$.

As portal pressure increased the capillary blood flow to the colon was reduced in dogs (5-12) (Fig. 2). A highly significant negative correlation between capillary flow and portal pressure was demonstrated by analysis of covariance $(F=289 ; 1,6$ degrees of freedom; $P<0.001)$. When capillary flow was plotted against superior mesenteric arterial flow for the same experiment (Fig. 3) the resulting correlation was again highly significant $(F=38 ; 1,6$ degrees of freedom; $P<0.001$ ).

Capillary blood flow to the colon was compared with that of other tissues at basal portal pressure in dogs (5-12) (Fig. 4). The kidney received by far the greatest relative capillary flow of any of the tissues studied. The colonic capillary flow was not significantly different from that of any of the other areas of bowel studied. There was no relationship between relative capillary flow to any of the tissues (except the colon) and either portal pressure or superior mesenteric arterial flow when adjustments to portal pressure were made.

\section{Discussion}

In general terms, previous studies ${ }^{3,4}$, have indicated that a relationship exists between small intestinal absorption and splanchnic blood flow. We have previously shown that both colonic absorption and mesenteric blood flow are related to portal pressure. ${ }^{1}$ The present study was designed to investigate colonic haemodynamics at the mucosal level.

The use of radio-labelled microspheres of approximately $15 \mu$ diameter has become a standard technique in the estimation of capillary blood flow. ${ }^{56}$ The size of the particles ensures that circulating microspheres will not pass through capillary beds but will pass through arteriovenous or portovenous anastomoses greater than $15 \mu$ in diameter. When a uniformly mixed quantity is present in the arterial blood, the number of microspheres retained by parallel-coupled tissues of the vascular bed is proportional to the capillary blood flow in the tissues. To ensure thorough mixing with the aortic blood, the microspheres were injected into the left ventricle after complete disaggregation in Tween 80 solution. The size of the three types of microspheres was very similar and any discrepancies caused by variation in size should be obviated by the fact that the order of injection was randomly varied when different types of microspheres were used in the same experiment.

When microspheres were injected at basal portal pressure (Fig. 4) the jejunum received the largest relative flow of any region of the intestine with a progressive fall along the small intestine. This is in agreement with the studies of Steiner and Muller ${ }^{7}$ who used ${ }^{86} \mathrm{Rb}$ fractionation in rats, $\mathrm{Geber}^{8}$ who directly measured total flow to different regions of the dog intestine with an electromagnetic flowmeter, and Bond et al. ${ }^{9}$ who used radio-labelled microspheres. The finding that the colon received a larger relative flow than the ileum, however, is not in agreement with previous studies. ${ }^{79}$ The capillary flow to the kidney was greater than to any other organ, as expected. ${ }^{10}$ The relative proportion of microspheres retained by the liver was lower than any other organ because a large proportion of its blood supply has previously passed through the mesenteric organs. Considerable variation existed between duplicate samples from different regions of all the organs studied which suggests that regional variations in capillary flow occur in those organs. It is suggested that an entire organ should be studied for accurate assessments of blood flow using microspheres. The standard errors of these results were large, due to variation between individual animals.

When the different regions of the colon are considered separately (Fig. 1). The proximal colon seems to receive a greater relative capillary flow than the distal region. This may be related to the greater absorptive capacity of the proximal colon compared with the remainder of the large bowel. ${ }^{11}$

At basal portal pressure, more than $90 \%$ of the capillary flow to the colon was to the mucosa, the submucosa and serosa receiving very small proportions of the flow. The distribution is similar to that found in the canine small intestine using $12 \mu$ microspheres ${ }^{5}$ or ${ }^{86} \mathrm{Rb}$ uptake methods, ${ }^{12}$ although higher proportionate serosal flows were found in these earlier studies. However, Greenway and Murthy ${ }^{13}$ reported a much larger submucosal flow in total intestine of the cat using radio-labelled microspheres.

At a high portal pressure $(35 \mathrm{mmHg})$ the distribution of blood flow to the three layers remained very similar. This suggests that short-term changes in splanchnic blood flow, produced by portal venous occlusion, are not associated with a shift in blood flow distribution within the colon. The results, however, do not exclude the possibility of redistribution within the mucosa-for example, from villous to non-villous mucosa. However, these results clearly show that, at normal and raised portal pressure, the blood flow to the colonic mucosa can be accurately deduced by measuring the total colonic capillary blood flow. 
When superior mesenteric arterial flow was altered by varying the portal pressure, we found an approximately linear relationship occurs between colonic capillary blood flow, and portal pressure on the one hand, and superior mesenteric arterial flow on the other (Figs. 2 and 3). As the capillary flow is largely to the mucosa at normal and raised portal pressure, it follows that mucosal capillary blood flow is proportional to total splanchnic blood flow over a wide range of portal pressures $(5-50 \mathrm{mmHg})$. There is no evidence to suggest that alterations in splanchnic blood flow produced by mechanical means are in any way affected by arteriovenous shunting mechanisms. As we have previously shown that colonic absorption of ammonia, water and electrolytes is related to portal pressure over a similar range of portal pressures, ${ }^{1}$ it follows that colonic absorption and mucosal capillary flow are similarly related.

Care must be taken in relating these results to chronic portal hypertension in patients because of species differences and the acute nature of the experiments. However, the results support the concept that sudden alterations in portal venous pressure-for example, after portacaval shunt-may produce immediate changes in colonic mucosal capillary flow resulting in altered rates of colonic absorption.

The authors wish to thank Miss A Birch for her technical assistance. The research was supported by a grant from the Mersey Regional Health Authority.

\section{References}

${ }^{1}$ Grandison AS, Harrison ID, Shields R. The effects of wide variations in portal pressure on mesenteric blood flow and absorption from the canine colon. Gut 1980; 21: 475-9.

${ }^{2}$ Snedecor GW, Cochran WG. Statistical methods. 6th ed. Iowa: State Univ Press, 1967.

${ }^{3}$ Pytkowski B, Michalowski J. Motility-and blood flowdependent absorption of amino acids in canine small intestine. Eur J Clin Invest 1971; 7: 79-86.

${ }^{4}$ Varro V, Blaho G, Csernay L. Effect of decreased local circulation on the absorptive capacity of a small intestine loop in the dog. Am J Digest Dis 1965; 10: 170.

${ }^{5} \mathrm{Grim}$ E, Lindseth EO. Distribution of blood flow to the tissues of the small intestine of the dog. Univ Minnesota Med Bull 1958 ; 30: 138-45.

${ }^{6} \mathrm{Greenway} \mathrm{CV}$, Oshiro G. Intra hepatic distribution of portal and hepatic blood flows in anaesthetized cats and dogs and the effects of portal occlusion, raised venous pressure and histamine. J Physiol 1972; 227: 473-85.

${ }^{7}$ Steiner SH, Mueller GCE. Distribution of blood flow in the digestive tract of the rat. Circ Res 1961; 9: 99102.

${ }^{8}$ Geber WF. Quantitative measurement of blood flow in various areas of small and large intestine. Am J Physiol 1960; 198: 985-6.

${ }^{9}$ Bond JH, Prentiss RA, Levitt MD. The effect of anesthesia and laparotomy on blood flow to the stomach, small bowel, and colon of the dog. Surgery 1980; 87: 313-8.

${ }^{10}$ Folkow B, Neil E. Circulation. Oxford University Press. 1971.

${ }^{11}$ McNeil NI, Cummings JH. Evidence for regional variation in large intestinal function. (Abstract). Gut 1979; 20 : A439.

${ }^{12}$ Ross G. Effects of epinephrine infusions on mesenteric arterial blood flow and its tissue distribution. Proc Soc Exp Biol Med 1971; 137: 921-4.

${ }^{13}$ Greenway CV, Murthy VS. Effects of vasopressin and isoprenaline infusions on the distribution of blood flow in the intestine; criteria for the validity of microsphere studies. Br J Pharmac 1972; 46: 177-88. 